\title{
Regulação em Saúde: desafios à governança do SUS
}

\author{
Health Regulation: challenges to the Unified Health \\ System governance
}

\author{
Dayse Vieira Santos Barbosa ${ }^{1}$, Nelson Bezerra Barbosa², Estela Najberg ${ }^{3}$
}

\begin{abstract}
Resumo
O Sistema Único de Saúde tem desenvolvido estratégias de racionalização do uso dos recursos, expressas no sistema de regulação. Nesse sentido, o desafio que o sistema enfrenta refere-se à capacidade de organizar as atividades desse sistema. Este artigo tem como objetivo descrever a política de regulação adotada no âmbito do SUS, considerando seus elementos conceituais e operacionais, com foco na atenção às urgências. Trata-se de um estudo descritivo, baseado em fontes secundárias. Toma como ponto de partida a definição de regulação, derivada da economia, e descreve o processo e instrumentos de estruturação de um sistema de organização da oferta e demanda em saúde. A definição de responsabilidades entre os gestores conforma um processo de organização de um sistema de governança direcionado à efetiva regulação do sistema, em especial para ordenação da oferta e demanda em uma de suas áreas mais sensíveis - a atenção às urgências e emergências. A estruturação e funcionamento da rede de serviços nessa área podem se beneficiar do controle do fluxo e da qualidade de serviços, dependendo da capacidade de uso dos elementos constitutivos do sistema de regulação, com ênfase na sua governança.
\end{abstract}

Palavras-chave: política de saúde; gestão em saúde; serviços médicos de emergência; regulação e fiscalização em saúde.

\begin{abstract}
The Unified Health System (SUS) has rationalized the use of resources, which is expressed in the regulation system. Thus, the challenge faced by the system refers to the ability of organizing its own activities. This article aims to describe the regulatory policy adopted by SUS, considering its conceptual and operational elements, focusing on the attention to the emergencies. This is a descriptive study, based on secondary sources. It takes as a starting point the definition of regulation, derived from Economics and describes the processes and tools for the structuring of an organization system of supply and demand in health. The definition of responsibilities among managers forms an organizing process of a governance system directed to an effective regulation, especially for ordering of supply and demand in one of its most sensitive areas - attention to urgency and emergency care. The structuring and operation of network services in this area can benefit itself from the flow control and from the services quality, depending on the usability of the constitutive components from the regulatory system, emphasizing its governance.
\end{abstract}

Keywords: health policy; health management; emergency medical services; health care coordination and monitoring.

${ }^{1}$ Curso de Medicina, Centro Universitário de Anápolis (UniEVANGÉLICA) - Anápolis (GO), Brasil.

${ }^{2}$ Faculdades Alfa - Goiânia (G0), Brasil.

${ }^{3}$ Faculdade de Administração, Ciências Contábeis e Ciências Econômicas (FACE), Universidade Federal de Goiás (UFG) - Goiânia (GO), Brasil.

Trabalho realizado na Secretaria Municipal de Saúde - Goiânia (GO), Brasil.

Endereço para correspondência: Dayse Vieira Santos Barbosa - Rua C-152, Q-384, L-11 - Jardim América - CEP: 74275-120 - Goiânia (G0), Brasil -

Email: daysevsbarbosa@hotmail.com

Fonte de financiamento: Fundação de Amparo à Pesquisa do Estado de Goiás (FAPEG).

Conflito de interesses: nada a declarar. 


\section{INTRODUÇÃO}

A transição demográfica e epidemiológica no Brasil, apontada em dados censitários ${ }^{1}$, evidencia o aumento da morbimortalidade por causas crônico-degenerativas e por causas externas (acidentes e violência) e, em consequência, a demanda por serviços de urgência.

Em decorrência dessa realidade, o Sistema Único de Saúde se tornou a principal referência para entrada dos casos de acidentes e violências, com registro de 1.123.335 internações no Sistema de Informações Hospitalares (SIH) e R \$ 1.298.377.954,54 de gastos hospitalares no ano de 2014 por essas causas².

Os óbitos por causas externas dizimam a população economicamente ativa, são precedidos de situações de urgência clínica e cirúrgica que incidem no perfil de morbidade e já são identificados entre os principais problemas de saúde pública no país. Interessa destacar que a evolução do perfil de morbimortalidade no Brasil e a transição demográfica, observadas no período anterior à criação do SUS, continuam presentes também atualmente nos indicadores que as dimensionam. Registros normativos retrospectivos ao SUS denotam um processo progressivo de reestruturação do sistema de serviços de saúde na perspectiva de organizar as ações direcionadas às urgências dentro de uma lógica sistêmica, conformando uma política de urgências ${ }^{3}$.

Com base no diagnóstico das necessidades de saúde numa determinada área geográfica e na definição de grades assistenciais de referência pactuadas entre gestores de suas unidades federadas, torna-se exequível organizar os sistemas regionais de saúde em suas especificidades (atenção às urgências, atenção à saúde materno-infantil e atenção à saúde do homem) e estabelecer protocolos e fluxos dos pacientes nos diferentes níveis de atenção de suas unidades componentes - o que denominamos Regulação.

A definição de responsabilidades entre os gestores configura um processo de organização de um sistema de governança no âmbito do SUS, voltado para a efetiva regulação do sistema, em especial para a organização da oferta e da demanda em uma de suas áreas mais sensíveis - a atenção às urgências e emergências.

Nesse sentido, o presente trabalho pretende descrever a política de regulação adotada no âmbito do SUS, com ênfase para sua importância na implementação da política de urgências. Para tanto, foram consultadas referências bibliográficas e documentais que tratam do tema, considerando aspectos conceituais e operacionais sobre o desenvolvimento dessa política no âmbito do Sistema Único de Saúde (SUS). Os descritores utilizados na consulta foram: política de saúde, regulação em saúde e redes de atenção em saúde.

\section{CONCEITUAÇÃO, FORMATO E ALCANCE DA REGULAÇÃO}

A regulação das urgências adveio da necessidade de se organizar e ordenar a oferta de ações e serviços na Rede de Atenção às Urgências (RAU), articulando os diferentes níveis de densidade tecnológica inerentes aos componentes da rede e dentro dela.

É necessário assinalar que a falta ou a dificuldade de acesso à assistência nos serviços de atenção primária, a baixa resolubilidade desse nível assistencial e a não viabilidade da contrarreferência do paciente para o acompanhamento ambulatorial induzem os usuários com queixas crônicas e sociais a procurar os serviços de urgência como porta de entrada do sistema de saúde.

Soma-se a isso a insuficiente oferta de leitos hospitalares nos serviços da rede assistencial, que prolonga a permanência dos pacientes em espera do leito apropriado para continuidade da assistência. De maneira geral, os leitos hospitalares de urgência disponíveis nos municípios-polo regionais não servem à assistência exclusiva dos seus munícipes. Oriundos de outros municípios e estados, pacientes chegam às unidades hospitalares de referência no atendimento às urgências, ora por livre demanda, ora encaminhados por mecanismos de pactuação entre municípios.

Dessa forma, as emergências estão superlotadas, comprometendo a agilidade e a qualidade da assistência, fato que ganha repercussão frequente na grande mídia, com expressão de descontentamento da população. São apontadas deficiências da estrutura física, da gestão e de recursos humanos nos serviços de urgência como fatores que têm causado insatisfação e insegurança dos profissionais e usuários ${ }^{4}$. Sobrepõem-se a essas dificuldades, a propensão ao agravamento dos quadros de urgência com progressão para a letalidade ou a maior incidência de sequelas.

Nesse contexto, torna-se compreensível a importância da implementação do processo de regulação das urgências, corporificado em políticas públicas, como mecanismo de organização da assistência com vistas a desconcentrar a atenção efetuada pelos prontos-socorros e contribuir para a limitação do dano na população assistida.

Uma das diretrizes da RAU colocadas na política de urgências é a regulação articulada entre todos os seus componentes, "com garantia da equidade e integralidade do cuidado". Outra diretriz, a de regionalização do atendimento às urgências, propõe "articulação das diversas redes de atenção e acesso regulado aos serviços de saúde"s. Introduz-se, aqui, uma nova lógica: a articulação na RAU e entre as redes e a regulação do acesso na linha de cuidado.

A Regulação Médica das Urgências permite que se responda a essa demanda concorde às diretrizes do SUS, de forma harmoniosa, proporcional e ordenada, “[...] consistindo o ato de regular em guiar, orientar, ajustar, sujeitando a regras 
todas as respostas às situações de urgência/emergência em um município ou região $[. . .]^{3}$.

$\mathrm{O}$ conceito de regulação deriva da Economia e tem como significado o controle sobre as falhas de mercado, observando-se os dois polos da relação em ambientes de mercado - a oferta e a demanda. O debate no campo de ação do setor saúde se inicia com o escopo dessa atividade (regulatória) voltada para o mercado de planos e seguros de saúde. No âmbito do SUS, ela visa ordenar a relação entre as necessidades dos usuários e a capacidade de oferta de sistemas e serviços ${ }^{7}$.

A saúde pode ser vista como um dos diversos setores da atividade econômica e, portanto, do mercado, que pode apresentar falhas a justificar a intervenção do Estado. Num sistema ideal, a oferta e a procura definem os preços dos bens e serviços que são produzidos e distribuídos em condições de perfeita competição. O equilíbrio é alcançado pela maximização de lucro pelos produtores e pela maximização da utilidade dos bens e serviços pelos consumidores ${ }^{7}$.

As condições para a perfeita competição são a racionalidade dos consumidores e produtores, a inexistência de externalidades, o perfeito conhecimento do mercado pelo consumidor, a capacidade de livre decisão pelo consumidor, em seu próprio benefício, e a impossibilidade de que os produtores influenciem o mercado. Entretanto, nenhuma das condições de perfeita competição está presente no setor saúde. A existência de riscos e incerteza é caracterizada pela imprevisibilidade da doença, que dificulta o planejamento de gastos futuros com saúde e pode levar ao desenvolvimento de mecanismos privados de seguro a ressarcir custos ${ }^{8}$.

O mercado de seguro privado de saúde aparentaria “[...] uma solução para responder ao problema da incerteza quanto ao futuro; no entanto, no mercado de seguro privado de saúde ocorrem também falhas"; citadas "[...] as deseconomias de escala, a seleção adversa e o risco moral [...]" (p. 126)7.

A "deseconomia de escala" decorre da competição entre pequenas companhias de seguro saúde, que repercute no maior custo final do seguro para o consumidor. Seu contrário - a economia de escala - representa menor custo por unidade de produto, como resultado da distribuição do custo fixo de grandes empresas 7 .

Já a "seleção adversa" consiste na exclusão do acesso ao seguro saúde por segurados como idosos e portadores de doenças crônicas, que se veem impossibilitados de custear o seguro relativo às situações de maior risco às quais estão submetidos ${ }^{7}$.

O "risco moral" consiste em excesso de demanda que aumenta preços por duas razões. Do lado do consumidor, a confiança no mecanismo de seguro de custo fixo leva à procura não controlada por serviços de saúde e diminui a preocupação com a manutenção da saúde individual, o que amplia o risco de doenças e a consequente procura por assistência. Do lado do provedor de serviços, o profissional da saúde, pode haver influência em aumento da procura na medida em que pode influenciar a decisão dos pacientes em prol de mais procedimentos do que os necessários - caracterizando indução da procura pela oferta ${ }^{7}$.

Essas falhas realçam que o mercado, por si só, não é capaz de promover o equilíbrio entre oferta e demanda - isso justifica a intervenção do Estado na regulamentação e produção de bens e serviços do setor saúde. A regulamentação também é necessária pela alta importância social que o setor detém, suscitando a necessidade de equidade, provavelmente pouco atingível apenas pelo equilíbrio de mercado.

O alcance da ação regulatória na área da saúde pública traduz-se no grupo de ações mediatas que se põem de permeio entre as demandas dos usuários e seu acesso aos serviços de saúde 9 . Esse significado da regulação incorpora competências de condução política, análise da situação, planejamento e comunicação, entre outras, na organização e controle dos fluxos de acesso, do desempenho dos sistemas e da qualidade da assistência.

Importante instrumento de ordenação dos serviços e ações de saúde, a regulação coaduna com os princípios regentes do SUS, pois pretende a universalidade e equidade na sua prestação; a descentralização com distribuição compartilhada de objetivos e compromissos entre as unidades federadas; e a regionalização e hierarquização da rede para a garantia da integralidade e continuidade do cuidado aos cidadãos em seu contexto geográfico, sociocultural e sanitário ${ }^{5}$.

A regulação é incluída pelos gestores na elaboração quadrianual dos Planos de Saúde e definida pela Organização Pan-Americana de Saúde ${ }^{10}$ como uma das Funções Essenciais de Saúde Pública (FESP) que expressam o papel gestor do Estado na saúde ${ }^{11}$.

Os processos regulatórios propiciam à gestão pública $\mathrm{o}$ estabelecimento de um melhor controle do acesso aos serviços ofertados e da aplicação dos recursos (eficiência), os quais favorecem a organização do sistema de saúde para a atenção às urgências (eficácia) e qualificam essa atenção de forma a proporcionar o alcance em maior dimensão dos objetivos sanitários coletivos propostos na política de saúde (efetividade) ${ }^{5}$.

Tendo o país dimensões continentais favorecedoras de diferenças e iniquidades regionais que repercutem na atenção à saúde, incluída a atenção às urgências, a regulação contribui com a diversidade operativa das singularidades regionais para superação dessas desigualdades.

\section{POLÍTICA NACIONAL DE REGULAÇÃO (PNR) DO SUS}

A instituição formal da PNR do SUS dá-se pela Portaria MS/GM n ${ }^{\circ} 1.559$, de $1^{\circ}$ de agosto de 2008, que toma regulação como "[...] conjunto de ações que facilitam ou limitam a produção 
de bens e serviços no setor saúde, por meio da regulamentação, controle, fiscalização, monitoramento, auditoria e avaliação [...]"12.

A referida norma classifica a regulação em três dimensões de atuação, integradas segundo as competências da gestão tripartite do SUS: Regulação de Sistemas de Saúde (RSS) - exercida sobre os sistemas. Regulação da Atenção à Saúde (RAtS) - exercida sobre a produção das ações de atenção à saúde nos diversos níveis de complexidade e Regulação do Acesso à Assistência (RAA) ou Regulação do Acesso ou, ainda, Regulação Assistencial - exercida sobre a organização, o controle, o gerenciamento e a priorização do acesso dos usuários aos serviços de saúde ${ }^{12}$.

Por estar intimamente relacionado à organização da atenção, o conceito de redes remete à regulação médica do fluxo e dos mecanismos de transferência de pacientes por centrais de regulação que, inseridas nos complexos reguladores, permitam o consumo racional dos recursos destinados às urgências, de modo a garantir o acesso aos recursos assistenciais de saúde, necessários para as pessoas e a população usuária.

Dessa forma, o sistema de regulação, na medida em que opera sobre a relação entre oferta e demanda das ações e serviços de saúde na perspectiva da racionalização dos gastos, tem papel importante na melhoria da gestão dos recursos financeiros e materiais do sistema.

\section{COMPLEXOS REGULADORES (CRg)/CENTRAIS DE REGULAÇÃO (CR)}

Os CRg são estruturas que operacionalizam as ações da regulação do acesso por meio da articulação e integração de um conjunto de CR: Central de Regulação de Urgências, Central de Internações Hospitalares e Central de Consultas e Exames. As CR são implantadas como unidades de trabalho dos CRg.

Considera-se importante assinalar que o preconizado na PNR corrobora o conceito dos CRg, anteriormente introduzido no Pacto de Gestão, e a sua operacionalização mediante protocolos assistenciais vinculados com a contratação, controle assistencial e avaliação, e com outras funções da gestão, como programação e regionalização, a fim de garantir a organização das redes e fluxos assistenciais ${ }^{13}$.

Outro fator representativo implícito na regulação é a responsabilização gestora pactuada e "delimitada por níveis de competência e complexidade da rede de serviços", que conduz ao estabelecimento de "fluxos de referência e contrarreferência segundo os Planos Diretores de Regionalização - PDR”, fundamentados na "necessidade demandada e na capacidade física instalada" dos entes federados. Uma vez definidas e pactuadas as "regras de referenciamento intraestaduais, intramunicipais e/ou interestaduais com a garantia do financiamento das ações por meio da Programação Pactuada e Integrada - PPI”, torna-se perceptível a necessidade de regulação desses fluxos nos espaços e funcionalidades dos $\mathrm{CRg}^{9}$.
Os CRg podem ter abrangência intramunicipal, municipal, micro ou macrorregional, estadual ou nacional ${ }^{9}$; ao passo que a PNR do SUS preconiza estruturas com modelos de CRg Estadual, CRg Regional e CRg Municipal, assim caracterizados:

I - Complexo Regulador Estadual: Gestão e gerência da Secretaria de Estado da Saúde, regulando o acesso às unidades de saúde sob gestão estadual e a referência interestadual e intermediando o acesso da população referenciada às unidades de saúde sob gestão municipal, no âmbito do estado.

II - Complexo Regulador Regional:

a) Gestão e gerência da Secretaria de Estado da Saúde, regulando o acesso às unidades de saúde sob gestão estadual e intermediando o acesso da população referenciada às unidades de saúde sob gestão municipal, no âmbito da região, e a referência inter-regional, no âmbito do estado;

b) Gestão e gerência compartilhada entre a Secretaria de Estado da Saúde e as Secretarias Municipais de Saúde que compõem a região regulando o acesso da população própria e referenciada às unidades de saúde sob gestão estadual e municipal, no âmbito da região, e a referência inter-regional, no âmbito do estado; e

III - Complexo Regulador Municipal: Gestão e gerência da Secretaria Municipal de Saúde, regulando o acesso da população própria às unidades de saúde sob gestão municipal.

Essas estruturas e suas práticas e processos de trabalho operacionalizam toda a arquitetura desenhada no processo de formulação e normatização da Política Nacional de Regulação. Atuam, portanto, como um centro nervoso que busca equalizar recursos disponíveis e demandados pelos usuários. A organização do fluxo de usuários dentro das redes e unidades de saúde problematiza, constantemente, a capacidade de resposta do sistema e informa sobre as dificuldades para sua legitimação junto à sociedade 9 .

A transação de dados proporcionados pelos sistemas de informação nas diversas unidades do sistema de regulação tem buscado dar maior racionalidade ao uso dos recursos e aportar ao usuário maior comodidade e celeridade nos encaminhamentos entre os pontos de atenção à saúde do SUS. Não é apenas um processo de substituição da "fila real" pela "fila virtual". A transposição de um espaço para outro fornece elementos para suporte ao processo de tomada de decisão no âmbito de articulação dos gestores, pela identificação dos tipos e forma de distribuição espacial das necessidades em saúde e dos investimentos correspondentes a serem feitos na ampliação da cobertura e na melhoria do acesso e da qualidade dos serviços prestados pelo SUS ${ }^{9}$.

\section{A INTEGRAÇÃO ENTRE AS POLÍTICAS DE REGULAÇÃO E DE ATENÇÃO ÀS URGÊNCIAS}

Considerando que a regulação sobre sistemas e serviços de saúde deve se dar sobre o modelo organizativo e a implementação deles, e que o SUS fez uma opção sobre uma organização 
de base territorial com foco na regionalização e na lógica de redes, o processo regulatório deverá observar esses princípios organizativos, integrando os diversos pontos de atenção disponíveis no território/região. As regiões de saúde se definem $\operatorname{como}^{14}$ :

[...] espaço geográfico contínuo constituído por agrupamentos de Municípios limítrofes, delimitado a partir de identidades culturais, econômicas e sociais e de redes de comunicação e infraestrutura de transportes compartilhados, com a finalidade de integrar a organização, o planejamento e a execução de ações e serviços de saúde.

As redes de atenção à saúde se caracterizam por serem organizações de serviços de saúde, sem vínculos de hierarquia entre si, que desenvolvem atenção integral à saúde de forma continuada a uma determinada população, em tempo oportuno e com qualidade suficiente para responder às suas demandas $\mathrm{e}$ necessidades. Organizam-se segundo a natureza específica do cuidado que devem desenvolver, sendo a atenção às urgências uma dessas configurações possíveis ${ }^{15}$.

As redes de atenção à saúde possuem três elementos constitutivos que fundamentam sua organização: população, estrutura operacional e modelo de atenção à saúde. A estrutura operacional tem centralidade no processo de integração entre as redes de atenção e o sistema de regulação, pois busca assegurar a oferta de ações e serviços de saúde.

A estrutura operacional da rede se desenvolve com base em um conjunto de elementos, quais sejam: o centro comunicador (Atenção Primária em Saúde), que deve coordenar os fluxos e contrafluxos dos cuidados; os pontos de atenção secundários e terciários (serviços especializados); os sistemas de apoio (diagnósticos e terapêuticos; de assistência farmacêutica; de informação em saúde); os sistemas logísticos (cartão do usuário, prontuário eletrônico, transporte; acesso regulado); e o sistema de governança.

Esse último se define como arranjos institucionais organizados que envolvem diferentes atores, estratégias e procedimentos, para gerir, de forma compartilhada e interfederativa, as relações entre as outras quatro estruturas operacionais citadas anteriormente, com vistas à obtenção de maior interdependência e melhores resultados sanitários e econômicos ${ }^{15,16}$.
Esse sistema tem como ponto sensível de seu desenvolvimento os centros reguladores, que devem produzir dados e informações sobre os diversos problemas de saúde de uma dada população, para apoio ao processo decisório sobre a implementação das ações de controle, inclusive o acesso a ações e serviços de saúde, entre esses os de urgência, que apresentam alto custo e impacto sobre o sistema de saúde.

\section{CONSIDERAÇÕES FINAIS}

A transição demográfica e epidemiológica representa um grande desafio ao processo de formulação, decisão e implementação de políticas públicas. Esse novo perfil da carga de doenças tem pressionado os setores de urgência e emergência, que operam com altos níveis de densidade tecnológica e são custos elevados para o sistema. A oferta de serviços nessa área reclama, portanto, uma maior racionalidade no uso dos escassos recursos do setor.

A integração entre as Políticas de Regulação e Atenção às Urgências representa, hoje, um enorme desafio na construção do sistema de governança para o SUS, pois impõe exigências em relação à expertise para desenvolvimento no âmbito da regulação, que abrangem: investimento em tecnologia da informação, para integrar e articular as bases de dados das várias centrais; capacidade de contratualização junto ao Sistema de Saúde Suplementar, no âmbito de cada região; provisão de recursos para investimentos na ampliação de serviços na rede pública; desenvolvimento de competências no campo da avaliação em saúde, com ênfase na capacidade resolutiva dos sistemas regionais; implementação de financiamento para atenção às urgências com foco no financiamento global e não no pagamento por procedimentos; desenvolvimento de projetos de corte intersetorial para redução dos riscos que incidem sobre a demanda na área de urgência.

A construção de sistemas regionalizados de saúde constitui lócus privilegiado para experimentação desse processo de integração entre essas duas políticas de controle das demandas na área de urgência e emergência, seus condicionantes e determinantes. O esforço inicial do sistema passa pelo desenvolvimento de competências para a resolução dos constrangimentos a essa integração, identificados acima.

\section{REFERÊNCIAS}

1. Instituto Brasileiro de Geografia e Estatística [Internet]. Brasília: IBGE; 2011 [citado em 2012 mar 26]. Disponível em: http://www.ibge.gov.br/ home/estatistica/populacao/estimativa 2011/ POP2011_DOU.pdf

2. Datasus. Informações em Saúde: Sistema de Informação Hospitalar [Internet]. Brasília; 2014 [citado em 2015 dez 30]. Disponível em: www. datasus.gov.br

3. Barbosa DVSB. Regulação médica do fluxo das urgências: análise do Complexo Regulador de Goiânia [dissertação]. Anápolis: Programa de Pós-graduação em Sociedade, Tecnologia e Meio Ambiente, Centro Universitário de Anápolis, UniEvangélica; 2012.

4. Jornal Medicina. Brasília: Conselho Federal de Medicina. Vol. 197, No. 26 2011.

5. Brasil. Ministério da Saúde. Portaria MS/GM n 1.600, de 07 de julho de 2011. Reformula a Política Nacional de Atenção às Urgências e institui a Rede de Atenção às Urgências no Sistema Único de Saúde (SUS). Diário Oficial da União, Brasília, 8 de julho de 2011. 
6. Rede Brasileira de Cooperação em Emergência [Internet]. 2011 [citado em 2012 fev 14]. Disponível em: http://www.rbce.org.br

7. Castro JD. Regulação em saúde: análise de conceitos fundamentais. Sociologias. 2002;4(7):122-135.

8. Donaldson C, Gerard K. Economics of health care financing: the visible hand. London: McMillan Press; 1993.. http://dx.doi.org/10.1007/978-1349-22512-5.

9. Brasil. Ministério da Saúde. Conselho Nacional de Saúde. Secretaria de Atenção à Saúde. Departamento de Regulação, Avaliação e Controle de Sistemas. Manual de implantação de complexos reguladores. Brasília; 2006.

10. Organização Pan-Americana de Saúde. Medição do desempenho das funções essenciais de saúde pública: guia para a aplicação do instrumento de desempenho das funções essenciais de saúde pública. Washington: OPAS; 2001.

11. Conselho Nacional de Secretários de Saúde. A gestão da saúde nos estados: avaliação e fortalecimento das funções essenciais. Brasília; 2007. 262 p.

12. Brasil. Ministério da Saúde. Secretaria de Atenção à Saúde. Portaria MS/GM $n^{\circ}$ 1559, de 1 de agosto de 2008. Institui a Política Nacional de Regulação do Sistema Único de Saúde. Diário Oficial da União, Brasília, 4 de agosto de 2008.

13. Brasil. Ministério da Saúde. Portaria $n^{\circ} 3.125$ de 7 de dezembro de 2006. Institui o Programa de Qualificação da Atenção Hospitalar de Urgência no Sistema Único de Saúde - Programa QualiSUS e define competências. Diário Oficial da União, Brasília, 8 de dezembro de 2006.

14. Brasil. Decreto ${ }^{\circ} 7.508$, de 28 de junho de 2011. Regulamenta a Lei 8.080 , de 19 de setembro de 1990. Diário Oficial da União [Internet], Brasília, 29 de junho de 2011 [citado em 2015 dez 30]. Disponível em: www.saude. gov.br

15. Mendes EV. As redes de atenção à saúde. Belo Horizonte: Escola de Saúde Pública; 2012.

16. Costa LMQ. Rede de Atenção ao Doente Renal Crônico: proposta de organização na lógica da Linha de Cuidado [tese]. Goiânia: Universidade Federal de Goiás; 2016. 